\title{
Role of Percutaneous Nephrostomy in Improving Quality of Life in Advanced Carcinoma Cervix Presenting with Obstructive Uropathy
}

\author{
Lynsel Texeira ${ }^{1}$, BH Santhosh Pai ${ }^{2}$, Nischith Dsouza ${ }^{3}$
}

\begin{abstract}
Aims and objectives: Percutaneous nephrostomy (PCN) is an interventional procedure which is mainly used in the decompression of the renal collecting system. Urinary diversion by this procedure can improve renal function. We examined the role of PCN in the management of advanced cervical cancer patients presenting with obstructive uropathy with a detailed analysis of improvement in the quality of life, morbidity, and survival rates.

Materials and methods: We prospectively evaluated 50 gynecology patients having cervical cancer with obstructive uropathy and deranged renal functions. A PCN was performed under local anesthesia using the Seldinger technique. Positions of catheters were confirmed with the nephrostogram at the end of the procedures. Health survey was conducted before the PCN procedure, 1st week, and 4th week and at the 3rd month after the PCN procedure.

Results: PCN was successfully established in all patients with the recovery of renal function. The median serum creatinine before and 1 month after the procedure was 6.6 and 2.0, respectively. Complications relating to the procedure (60\%) were fever/sepsis 7 (14\%), bleeding in 5 (10\%), pericatheter leak in $10(20 \%)$, and slippage of catheter requiring replacement in $7(14 \%)$. Overall, $45(90 \%)$ patients died of the primary disease and 5 are alive. The 3, 6, 9, and 12 months' death rates were $8(16 \%), 15(30 \%), 21(42 \%)$, and $2(1 \%)$, respectively. Fifty percent of death occurred within 6 months and $90 \%$ of death occurred by 9 months.

Conclusion: PCN provides a significant improvement in renal function parameters in majority of the patients. The quality of life is improved significantly at 1 week and 1 month which is not sustained at 3 months. As the benefits of PCN urinary diversion after 3 months are not established, counseling is essential and the wishes of the patient and her family have to be considered.

Keywords: Cervical cancer, Obstructive uropathy, Percutaneous nephrostomy.

Journal of South Asian Federation of Obstetrics and Gynaecology (2019): 10.5005/jp-journals-10006-1657
\end{abstract}

\section{INTRODUCTION}

Cervical cancer is the most common cancer in females. The incidence of cervical cancer in India is about 32 per 100,000 women. ${ }^{1}$ Mortality due to cervical cancer is also an indicator for death in $86 \%$ of people. ${ }^{2}$ Over $70 \%$ of the cases present in advanced stages of the disease with associated poor prognosis and high mortality rates. ${ }^{3}$ In many of them, it is difficult to offer definitive treatment as they present in uremia due to associated obstructive uropathy.

Urinary diversion by PCN is the commonly practiced procedure which can improve the renal function ${ }^{4}$ and also the quality of life. This would enable patients to tumor-specific treatment in majority of the cases and curative treatment in some of them. There are no prospective studies in literature to see if any urinary diversion improves the quality of life of these patients. There are also ethical issues like whether any intervention has to be done or not in terminal cases. There are no clear-cut guidelines for PCN in patients with advanced cervical cancer. The results of PCN are unpredictable in terms of recovery of renal functions, quality of life, and benefits attained to administer the subsequent palliative cure. Most studies reported in literature are retrospective, small sample based, and nonrandomized. In this study, we examined the role of PCN in the management of advanced cervical cancer patients presenting with obstructive uropathy with a detailed analysis of improvement in the quality of life, morbidity, and survival rates. The objective of the study is to find the quality of life before and after PCN using the SF 12 score and to access the intraoperative mortality,
${ }^{1}$ Department of Obstetrics and Gynecology, Kasturba Medical College, Mangaluru, Manipal Academy of Higher Educanon, Manipal, Karnataka, India

${ }^{2}$ Department of Nephrology, Yenepoya Medical College, Mangaluru, Karnataka, India

${ }^{3}$ Department of Urology, Yenepoya Medical College, Mangaluru, Karnataka, India

Corresponding Author: BH Santhosh Pai, Department of Nephrology, Yenepoya Medical College, Mangaluru, Karnataka, India, Phone: +91 9449471243, e-mail: drbhspai22@vahoo.co.in

How to cite this article: Texeira L, Pai BHS, et al. Role of Percutaneous Nephrostomy in Improving Quality of Life in Advanced Carcinoma Cervix Presenting with Obstructive Uropathy. J South Asian Feder Obst Gynae 2019;11(2):107-109.

Source of support: Nil

Conflict of interest: None

number of patients who can receive adjuvant treatment post-PCN, postoperative complications, and survival rate.

\section{Materials and Methods}

We prospectively evaluated 50 patients of gynecology patients having cervical cancer with obstructive uropathy and deranged renal functions that were referred during November 2010October 2016. The patients underwent urinary diversion by PCN 
at Yenepoya Medical College Hospital, Mangaluru. All cases of advanced carcinoma cervix were confirmed by biopsy. In all cases, the prognosis, subsequent treatment possibilities and expected results were explained in detail written informed consent was taken. The prognosts, subsequent treatment possibilities, and expected results were explained to individual participants.

The patients with confirmed advanced carcinoma cervix with obstructive uropathy (uremic symptoms with ultrasound/CT scan showing bilateral hydroureteronephrosis) with serum creatinine above $1.5 \mathrm{mg} / \mathrm{dL}$ and patients with carcinoma cervix who gave consent for PCN were included. Patients who presented with bilateral hydroureteronephrosis not primarily due to advanced carcinoma cervix (obstructive uropathy secondary to radiotherapy/ chemotherapy are excluded), patient with coagulopathy, and patients with advanced carcinoma cervix in whom retrograde stenting is possible were excluded.

The patients were followed up regular weekly hospital visits after discharge, during the first month and, thereafter, every monthly visit lifelong. Patients were dialyzed before the diversion if they had hyperkalemia or pulmonary edema. A PCN was performed under local anesthesia under C-arm/USG guidance using the Seldinger technique either bilateral or unilateral depending on the grades of hydronephrosis. Positions of catheters were confirmed with the nephrostogram at the end of the procedures. Renal function tests (RFTs) were measured on days 2 and 7, 1st month, and at the third month after PCN. Quality Metric's SF-12v2 ${ }^{\circledR}$. Health survey was conducted before the PCN procedure, 1st week, and 4th week, and at the third month after the PCN procedure.

Several variables were investigated and parameters affecting the quality of life were assessed. The paired $t$ test was applied to compare the quality of life before and after the PCN procedure. A $p$ value $<0.05$ was considered as statistically significant. All data were analyzed using the SPSS software (ver 17.0).

\section{Results}

Table 1 indicates the clinical characteristics of the patients. The mean age was 48 years. PCN was successfully established in all patients with the recovery of renal function. The median serum creatinine before and 1 month after the procedure was 6.6 and 2.0, respectively. Day 2 serum creatinine (DSCr) and 1st week serum creatinine (WSCr) showed a statistically significant reduction $(p=000)$ when compared with pre-PCN serum creatinine (PSCr).

Complications relating to the procedure (60\%) were fever/ sepsis 7 (14\%), bleeding in 5 (10\%), pericatheter leak in 10 (20\%), and slippage of catheter requiring replacement in 7 (14\%) (Table 2)

Overall, $45(90 \%)$ patients died of the primary disease and 5 are alive. The $3,6,9$, and 12 months' death rates were $8(16 \%)$, $15(30 \%), 21(42 \%)$, and 2 (1\%), respectively. Fifty percent of death occurred within 6 months and $90 \%$ of death occurred by 9 months. A difference in the quality of life was statistically significant between pre-PCN and post-PCN at 1 week and 1st month only (Table 3).

\section{Discussion}

Obstructive uropathy with uremia with the risk of impending irreversible renal damage is a common presentation in a significant proportion of cervical cancer patients in developing countries. ${ }^{5-7}$ $\mathrm{PCN}$ is useful especially in this situation, since retrograde ureteric stenting is often not possible. ${ }^{8}$ In the present study, $\mathrm{PCN}$ was feasible in all the 50 patients (100\%). Very low failure rates of $0-3 \%$ have been reported in other studies too. The present study with PCN
Table 1: Clinical characteristics of patients who underwent PCN

\begin{tabular}{ll}
\hline Median patient age (range) & \multicolumn{1}{l}{$48(30-66)$} \\
No. laterality of PCN procedure (\%) & $11(22)$ \\
Lt & $11(22)$ \\
Rt & $26(56)$ \\
Bilateral & $6.6(4.10-19.0)$ \\
Median mg/dL serum creatinine value (range) \\
Preoperative & $2.5(1.80-7.80)$ \\
Postoperative (1st week) & $2.0(1.0-7.80)$ \\
Postoperative (1st month) & $8.6(6.6-13.5)$ \\
Median g/dL of hemoglobin & \\
before PCN (range) & \\
Quality of life (percentage) & $35(30-45)$ \\
Pre-PCN & $49(32-56)$ \\
1st week post-PCN & $52(42-56)$ \\
1st month post-PCN & $42(29-56)$ \\
3rd month post-PCN & \\
PCN under (\%) & $5(10)$ \\
USG guidance & $45(90)$ \\
C-Arm guidance & \\
PCN catheter size (\%) & $7(14), 19(38), 18$ (36), and 6 (12) \\
8, 10, 12, and 14 & \\
Hemodialysis (\%) & $42(84)$ \\
1-4 & $4(8)$ \\
4-6 & \\
Nill & \\
Post-PCN palliative procedures (\%) & \\
RT & \\
CT & \\
Stenting & \\
None & \\
\hline & \\
&
\end{tabular}

Table 2: Complications relating to the procedure in the present study

\begin{tabular}{lrc}
\hline Complications & No. & $\%$ \\
\hline Bleeding & 5 & 10 \\
Fever/sepsis & 7 & 14 \\
Pericatheter leak & 19 & 20 \\
Slippage and replacement & 7 & 14 \\
Others & 1 & 2 \\
\hline
\end{tabular}

Table 3: Difference in quality of life between pre-PCN and post-PCN

\begin{tabular}{lccccc}
\hline \multirow{2}{*}{$\begin{array}{l}\text { Paired samples } \\
\text { (quality of life) }\end{array}$} & \multicolumn{5}{l}{ Paired differences } \\
\cline { 2 - 6 } & Mean & SD & SE & t value & p value \\
\hline $\begin{array}{l}\text { Pre-PCN and 1st week } \\
\text { post-PCN }\end{array}$ & 13.90 & 5.59 & 0.7914 & 17.564 & 0.000 \\
$\begin{array}{l}\text { Pre-PCN and 1st month } \\
\text { post-PCN }\end{array}$ & 17.44 & 8.10 & 1.146 & 15.207 & 0.000 \\
$\begin{array}{l}\text { Pre-PCN and 3rd month } \\
\text { post-PCN }\end{array}$ & 11.41 & 15.43 & 2.276 & 2.0514 & 0.219 \\
\hline
\end{tabular}

and manageable complications were noted in $60 \%$ cases, almost the same as reported in other studies $52-83 \%$. Literature reports cited $29-60 \%$ of incidence of reinsertion. ${ }^{6,9}$ It was noted in $14 \%$ of the cases in the present study.

Romera et al. showed that PCN is more useful in cervical cancer patients than those of bladder/prostrate cancers with obstructive uropathy with prolonged survival. ${ }^{10}$ Our study showed that the 
1-year survival rate is around $6 \%$. In a small but definite number of cases, palliative treatment with a long survival (in months) could be achieved. Though the quality of life pre- and post-PCN (1st week and 1st month) was statistically significant, the quality of life did not improve in the long-term (third month).

\section{Conclusion}

PCN provides a significant improvement in renal function parameters in majority of the patients with advanced cervical cancer presenting with obstructive uropathy. There is no procedure-related mortality, with minimal morbidity. The quality of life is improved significantly at 1 week and 1 month which is not sustained at the third month. As we did not have a comparable group without PCN, our study cannot ascertain the survival advantage with diversion (PCN), which can be done by comparing a similar cohort of patients who did not receive diversion. As the benefits of PCN urinary diversion after 3 months are not established, counseling is essential and the wishes of the patient and her family have to be considered.

\section{References}

1. National Cancer Registry Programme, Annual Report, ICMR, New Delhi: 2005.
2. Satija A. Cervical cancer in India. South Asia centre for chronic disease. [Accessed February 16, 2014].

3. Dinshaw KA, Rao DN, et al. Tata Memorial Hospital Cancer Registry Annual Report, Mumbai, India; 1999.

4. Massry SG, Schainuck LI, et al. Studies on the mechanism of diuresis after relief of urinary obstruction. Ann Intern Med 1967;66:149-158. DOI: 10.7326/0003-4819-66-1-149.

5. Halle MP, Toukep LN, et al. The profile of patients with obstructive uropathy in Cameroon: case of the Douala General Hospital. Pan Afr Med J 2016;23(1):67. DOI: 10.11604/pamj.2016.23.67.8170.

6. Mishra K, Desai A, et al. Role of percutaneous nephrostomy in advanced cervical carcinoma with obstructive uropathy: a case series. Indian J Palliat Care 2009 Jan;15(1):37. DOI: 10.4103/0973-1075.53510.

7. Chevalier RL, Forbes MS, et al. Ureteral obstruction as a model of renal interstitial fibrosis and obstructive nephropathy. Kidney Int 2009 Jun 1;75(11):1145-1152. DOI: 10.1038/ki.2009.86.

8. Van der Meer RW, Weltings S, et al. Antegrade Ureteral Stenting is a Good Alternative for the Retrograde Approach. Curr urol 2016;10(2):87-91. DOI: 10.1159/000447157.

9. Cohen EP, Sobrero M, et al. Reversibility of long-standing urinary tract obstruction requiring long-term dialysis. Arch Intern Med 1992 Jan 1; 152(1):177-179. DOI: 10.1001/archinte.1992.00400130177023.

10. Romero FR, Broglio $M$, et al. Indications for percutaneous nephrostomy in patients with obstructive uropathy due to malignant urogenital neoplasias. Int Braz J Urol 2005;31:117-124. DOI: 10.1590/ S1677-55382005000200005. 